\title{
Correlating bacterial shedding with fecal (a) CrossMark corticosterone levels and serological responses from layer hens experimentally infected with Salmonella Typhimurium
}

\author{
Pardeep Sharma, Vivek V. Pande, Talia S. Moyle, Andrea R. McWhorter and Kapil K. Chousalkar*
}

\begin{abstract}
Salmonella Enteriditis and Salmonella Typhimurium are commonly isolated during egg-related outbreaks of salmonellosis and represent a significant international public health issue. In Australia, Salmonella Typhimurium is the most common serovar identified in egg product related foodborne outbreaks. While a number of studies have investigated Salmonella shedding and host responses to infection, they have been conducted over a short time period. The present study sought to characterise bacterial shedding and host responses to infection in hens infected with only Salmonella Typhimurium or co-infected with both Salmonella Typhimurium and Salmonella Mbandaka over a 16 week period. Salmonella shedding was quantified using the most probable number and qPCR methods and was highly variable over the course of the experiment. On day 1, fecal corticosterone metabolites in birds infected with Salmonella Typhimurium (674.2 $\pm 109.3 \mathrm{pg} / \mathrm{mg})$ were significantly higher than control $(238.0 \pm 12.62 \mathrm{pg} / \mathrm{mg})$ or co-infected $(175.4 \pm 8.58 \mathrm{pg} / \mathrm{mg})$ birds. The onset of lay occurred between weeks $6-8$ post-infection (pi) and Fecal corticosterone metabolite (FCM) concentrations increased in both control and co-infected birds. Antibody responses to infection were monitored in both serum and yolk samples. Salmonella Typhimurium specific antibody was lower in co-infected animals than monoinfected animals. Bacterial loads in internal organs were characterised to determine persistence. Spleen, liver and caecal tonsils were positive for bacteria in both groups, indicating that Salmonella was not cleared from the birds and internal organ colonization could serve as a reservoir for continued bacterial shedding.
\end{abstract}

\section{Introduction}

Commercial poultry are often persistently infected with non-typhoidal serovars of Salmonella enterica. Eggs and raw egg based food products are often identified as the source of Salmonella during outbreaks of human gastrointestinal disease [1]. Thus, the zoonotic potential of Salmonella represents a significant global public health concern. In North America and Europe, the most common serovar isolated during egg-related outbreaks is Salmonella Enteritidis followed by Salmonella Typhimurium [2]. Strains of Salmonella Typhimurium, however,

*Correspondence: Kapil.chousalkar@adelaide.edu.au School of Animal and Veterinary Sciences, The University of Adelaide, Roseworthy, SA 5173, Australia are most frequently identified during Australian outbreaks of egg-related cases of salmonellosis [1].

Over the past several years, the incidence of human cases of salmonellosis in Australia has been increasing. In 2011, the total number of food related disease outbreaks had increased to over 150 and $38.4 \%$ were attributed to Salmonella [1]. Over the same period, the number of cases linked directly with eggs increased from 20.8 to $44.8 \%$ [1]. Despite improvements of on-farm control strategies, Salmonella Typhimurium remains a significant problem within the Australian layer industry [3].

Due to the public health importance of contaminated eggs, understanding the dynamics of Salmonella Typhimurium shedding patterns and associated host responses to infection is of critical importance. Previous experimental infection trials have examined 
egg contamination and internal organ colonization of layer hens. These studies, however, have infected birds at different ages, using a variety of inoculation methods [4-7] limiting the degree to which the data can be directly compared. Moreover, the data obtained from these investigations was collected for 3-4 weeks pi. The productive lifetime of a layer hen, however, can extend beyond 50 weeks of age and few studies have investigated extended bacterial shedding dynamics, egg contamination and host responses to infection.During productive lifespan, layer hens may also experience many physiological and environmental stressors, such as overcrowding, extreme temperature variation and the onset of lay that may lead to increased fecal shedding of Salmonella [811]. Stress has also been linked with impaired immunity $[8,9,12,13]$ which may increase intestinal colonization by enteric pathogens such as Salmonella [14]. The host immune response to Salmonella infection may also contribute to increased corticosterone levels however, relationship between persistent Salmonella colonisation and stress in birds is unclear.

In the Australian egg industry, Salmonella Typhimurium is frequently isolated from eggshell surfaces but it is not the only serovar isolated from egg farms $[15,16]$. The poultry farm environment is often contaminated with multiple serovars [15-17]. Field epidemiological investigations suggested that Salmonella Mbandaka was commonly isolated along with Salmonella Typhimurium in layer flocks without any clinical signs in chickens [16, 18]. Salmonella Mbandaka has not been associated with any egg related outbreaks in Australia [19], although this serovar has been associated with egg product related $\mathrm{Sal}$ monella outbreaks in the US [20].

Competition between co-infecting strains may affect the dynamics of one or more serovars. Layer hens environmentally infected with Salmonella Kentucky, for example, mitigated Salmonella Enteritidis colonisation of internal organs [21]. In addition, coinfection of layer hens with Salmonella Enteritidis, Salmonella Gallinarum and Salmonella Isangi has recently been shown to enhance disease in infected birds [22]. To date, there have been limited studies investigating how co-infection affects the dynamics of Salmonella Typhimurium shedding as well as host responses to infection.

Our hypothesis was that Salmonella Mbandaka can affect the shedding of Salmonella Typhimurium and internal organ colonization. We have conducted a 16 week infection trial, using layer hens reared free from exogenous Salmonella. Results from a companion study demonstrated that over the 16 week infection period, bacterial shedding was variable and that vertical transmission of Salmonella Typhimurium DT9 into egg internal did not occur [23]. The aims of the present study were to correlate fecal shedding and egg contamination patterns with host responses to infection (single and mixed) including fecal corticosterone levels as a marker of the host stress response as well as levels of Salmonella Typhimurium specific antibodies in the serum and yolk. A final aim of this study was to characterise persistence of Salmonella infection in peripheral organs.

\section{Materials and methods \\ Birds}

Fertile eggs were obtained from a commercial brown layer flock hatchery. Eggs were fumigated using formaldehyde and incubated for 21 days. A total of 32 pullets were hatched and raised in floor pens in positive pressure rooms within an animal housing facility located on the Roseworthy Campus of the University of Adelaide. The rooms within this facility, all animal cages, trays, and feeders had previously been cleaned and decontaminated using FoamCleanS and SaniGuard (Chemtall, Australia). At 10 weeks of age, birds were divided into three treatment groups: control $(n=4)$, Salmonella Typhimurium $(n=14)$ and Salmonella Typhimurium + Salmonella Mbandaka $(n=14)$ and housed individually in cages in separate rooms. Fumigated feed and sanitised water (Aquatabs, Ireland) were provided ad libitum to all birds. Feed, water and fecal samples were screened for Salmonella fortnightly by culture method as described previously [16]. This experiment was performed according to the Australian Code for the Care and Use of Animals for Scientific Purposes and was approved by the University of Adelaide Animal Ethics Committee (approval number: S-2014-008).

\section{Bacterial isolates}

Single isolates of Salmonella Typhimurium definitive type 9 (DT9) and Salmonella Mbandaka were used in this study. These Salmonella had been previously isolated from samples collected from layer hen farms during a previous epidemiology study [16] and serotyped at the Salmonella Reference Laboratory, Institute of Veterinary Medical Science (IMVS), Adelaide, South Australia.

\section{Challenge experiment}

At 14 weeks of age, just prior to lay, hens were orally inoculated with $1 \times 10^{9}$ colony forming units (CFU) of either Salmonella Typhimurium DT9 or a combination containing equal amounts of both Salmonella Typhimurium DT9 and Salmonella Mbandaka $\left(5.0 \times 10^{8} \mathrm{CFU}\right.$ of each serovar) suspended in Luria-Bertani (LB) broth (Oxoid, Australia). Serial tenfold dilutions of the inoculum were prepared and plated onto nutrient agar to confirm the total number of bacteria. Control birds received a sham inoculum containing only sterile LB broth. Clinical signs 
of infection were recorded throughout the experiment. At 30 weeks of age, [16 weeks post-infection (pi)] all birds were euthanized with Lethabarb (Virbac, Australia). Bone marrow, spleen, liver and caecal tonsils were collected from each bird for bacteriological examination.

\section{Enumeration of Salmonella in fecal samples}

A total of 320 fecal samples from individual hens were collected aseptically using sterile plastic bags on day 1 post-infection (pi) followed by $1,2,4,6,8,10,12,14$ and 16 weeks pi. Salmonella enumeration using the three tube most probably number (MPN) method was performed on all faecal samples as described previously [24]. Salmonella suspected samples were streaked onto xylose lysine deoxycholate (XLD) agar plates (Oxoid, Australia) and Salmonella Brilliance agar plates (Oxoid, Australia) for confirmation of Salmonella spp.

\section{Bacterial DNA extractions from fecal samples, egg shell wash and internal organs}

DNA was extracted from fecal samples using the Isolate Fecal DNA Kit (Bioline, Australia) following manufacturer instruction. DNA extraction from eggshell washes (enriched in RVS broth) collected from both infection groups was performed using Chelex ${ }^{\circledR}$ (Bio-Rad, Sydney, NSW, Australia) [25] The Wizard genomic DNA purification kit (Promega, Australia) was used to extract DNA from the tissue samples as per manufacturer instructions.

\section{Standard curve and qPCR for fecal samples for Salmonella Typhimurium and Salmonella Mbandaka}

The PCR detection of Salmonella was performed using the Quantifast ${ }^{\circledR}$ SYBER $^{\circledR}$ Green qPCR kit (Qiagen, Australia) in a total reaction volume of $10 \mu \mathrm{L}$ containing $2 \mu \mathrm{L}$ sample $(5 \mathrm{ng} / \mu \mathrm{L}), 5 \mu \mathrm{L}$ of $2 \times$ Quantifast SYBER Green Master Mix and $1 \mu \mathrm{M}$ of reverse and forward primers. Salmonella Typhimurium serovar specific primers TSR3 were used to detect Salmonella Typhimurium DT9. Further, to differentiate Salmonella Mbandaka from Salmonella Typhimurium DT9 in the co-infection group, primers for class 1 integron were used to specifically detect Salmonella Mbandaka [26]. The qPCR conditions were $5 \mathrm{~min}$ of denaturation at $95{ }^{\circ} \mathrm{C}$, followed by 40 cycles of denaturation at $95{ }^{\circ} \mathrm{C}$ for $10 \mathrm{~s}$ and $60{ }^{\circ} \mathrm{C}$ for $30 \mathrm{~s}$ each. Rotor-gene 1.7.75 (Corbett Research, Qiagen, Australia) software version was used for the data analysis. A standard curve was generated to establish the limit of detection and quantification of positive samples, by determining a serial tenfold dilution of spiked fecal samples with known concentrations of Salmonella Typhimurium or Salmonella Typhimurium + Salmonella Mbandaka.

\section{Fecal corticosterone analysis}

Fecal samples collected at day 1 (pi) followed by 1, 2, 4, $6,8,10,12,14$ and 16 weeks pi were thawed, mixed, and dried at $103{ }^{\circ} \mathrm{C}$ overnight. After cooling to room temperature, samples were ground to a fine powder. Corticosterone metabolites were extracted using methods recommended by the DetectX Corticosterone EIA kit manufacturer (Arbor Assays, Ann Arbor, USA). The concentration of fecal corticosterone metabolites (FCM) was measured by DetectX Corticosterone EIA kit as per manufacturer instruction.

\section{Survey of egg shell and egg internal contents for Salmonella contamination}

Eggs laid daily during 6, 8, 10, 12 and 14 weeks pi were collected and processed for Salmonella detection from both the eggshell and internal contents (Total eggs: 892; Control $=118$, Salmonella Typhimurium only $=365$, Salmonella Typhimurium + Salmonella Mbandaka coinfection $=409$ ) using previously described methods [16]. Eggshell wash enriched in Rappaport-Vassiliadis broth (RVS; Oxoid, Australia) was stored in $80 \%$ glycerol at $-80{ }^{\circ} \mathrm{C}$ to differentiate between Salmonella Typhimurium DT9 and Salmonella Mbandaka by standard PCR.

\section{PCR for egg shell wash and internal organ samples for Salmonella Typhimurium and Salmonella Mbandaka} Salmonella positive eggshell wash and internal organ samples from both infection groups were screened for the amplification of $i n v A$ and TSR3 gene for detection of Salmonella Typhimurium by multiplex PCR [26]. TSR3 gene was not amplified in Salmonella Mbandaka isolates [26]. Samples from both groups were also tested for the presence of Salmonella Mbandaka.

\section{Bacteriology of internal organs}

Bone marrow, spleen, liver and caecal tonsils were collected at week 16 pi and processed for bacteriology. Briefly, 0.1-0.2 grams of tissue sample were homogenised and serial tenfold dilutions were prepared in phosphate buffer saline (PBS). One hundred micro litre of each dilution was spread onto XLD agar plates and incubated overnight at $37^{\circ} \mathrm{C}$. After $24 \mathrm{~h}$, the bacterial colonies were enumerated and the number of Salmonella in tissues was expressed as mean $\log _{10} \mathrm{CFU} / \mathrm{g}$ of tissue.

\section{Serum and egg yolk sample collection and serologic examination by ELISA}

On day 0 and at $1,2,4,6,8,10,12$, and 14 weeks pi, $2 \mathrm{~mL}$ blood samples were collected from each bird and placed into serum clot activator tubes (Vacuette ${ }^{\circledR}$ tube, Greiner Bio-One, Australia). A total of 145 (Control; $n=20$, 
Salmonella Typhimurium only; $n=57$, Co-infection group; $n=68$ ) egg samples collected at weeks $6,8,10$, 12 and 14 pi were processed for the antibody extraction from the yolk samples. Egg yolk antibodies were extracted as described previously [27]. Dilutions of chloroform-extract egg yolk antibody were prepared from the pools of known positive and known negative eggs from control birds. Samples were tested in duplicate for the following dilutions; 1:10, 1:50, 1:100, 1:500 and 1:1000. From the curve produced, the linear part was expanded. Readings of known positive and negative samples individually at the selected dilution produced a cut-off value for the test. Threshold value were determined by plotting sensitivity and specificity against the cut off value using two graph receiver operating characteristics (TG-ROC) analysis as described [28]. A dilution factor of 1:100 was selected because it was on the linear part of the standard curve.

Antibody detection from both serum and egg yolk samples was tested using the Chicken Salmonella Typhimurium Antibody Kit LPS Group B (BioChek, Holland) and antibody titres were calculated according to manufacturer instruction.

\section{Statistical analysis}

The data for average $\log _{10} \mathrm{CFU} / \mathrm{qPCR}$, corticosterone level, and serum and egg yolk was analyzed using a two way analysis of variance (ANOVA) followed by a Tukey's multiple comparison of the mean. Significance between bacterial titres in organs was tested using a Mann-Whitney test. The correlation between MPN/g fecal count and Salmonella positive eggshell wash, average $\log _{10}$ CFU/ qPCR and corticosterone concentration was determined by Pearson correlation test ( $\mathrm{r}^{2}$ value). All data was analysed using either by GraphPad Prism version 6 software or IBM ${ }^{\circledR}$ SPSS Statistics ${ }^{\circledR}$ version 21. $p$ values $<0.05$ were considered statistically significant. A D'Agostino-Pearson omnibus normality test was conducted for all data. Serum and egg yolk antibody titres were normally distributed. MPN data were not normally distributed. MPN data was analysed by a Kruskal-Wallis with a Dunn's comparison of the means.

\section{Results}

Shedding and viable bacterial counts of Salmonella in fecal samples

Bacterial shedding varied significantly over time $(p<0.01)$ in both experimental treatment groups (Figure 1). The greatest number of viable bacteria observed in birds infected with only Salmonella Typhimurium occurred during week $1 \mathrm{pi}$, with a mean MPN/g of $48.53 \pm 16.55$. Samples collected from the Salmonella Typhimurium infection group in week 10 exhibited the

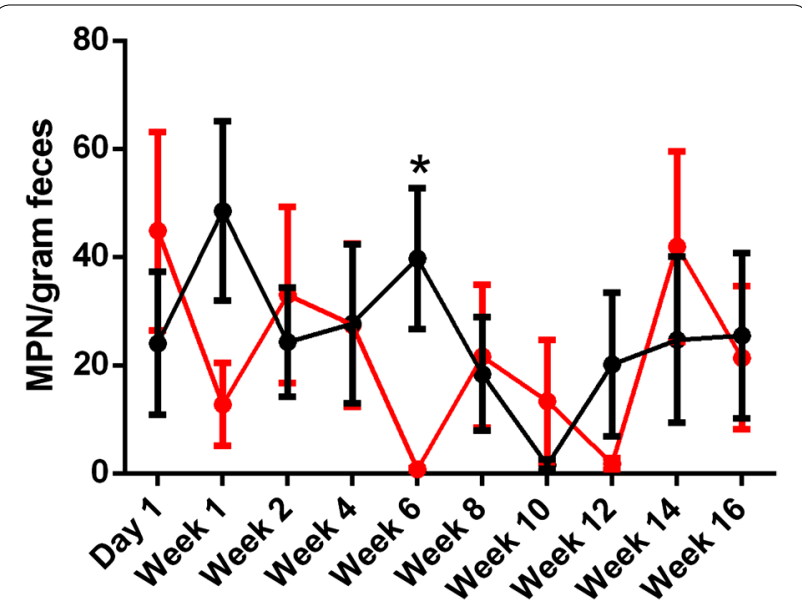

Figure 1 Enumeration of Salmonella in feces using most probable number (MPN) method. Fecal samples were collected from birds orally infected with $1 \times 10^{9} \mathrm{CFU}$ of Salmonella Typhimurium (Black line) or a combination of both Salmonella Typhimurium + Salmonella Mbandaka ( $5 \times 10^{8} \mathrm{CFU}$ of each serovar) (Red line) on day 1 and weeks $1,2,4,6,8,10,12,14$ and 16 pi. Data is presented as mean MPN/gram feces \pm standard error of the mean. Bacterial shedding in both infection groups varied significantly over the course of the experiment $(p<0.01)$. At week 6 pi, bacterial shedding was significantly higher in birds infected with only Salmonella Typhimurium group $\left({ }^{*} p<0.05\right)$

lowest mean MPN/g, $1.535 \pm 1.05$. For birds infected with both Salmonella Typhimurium and Salmonella Mbandaka, the greatest number of viable Salmonella was detected on day 1 pi with a mean MPN/g of $44.80 \pm 18.30$. The lowest mean MPN/g, $0.78 \pm 0.27$, was observed in the multi-serovar infection group at week 6 pi.

Over the entire experiment, no significant effect of time or treatment was detected between single and multi-serovar treatment groups $(p>0.05)$. At week $6 \mathrm{pi}$, however, birds infected with only Salmonella Typhimurium exhibited a significantly greater mean MPN/g than birds infected with both Salmonella Typhimurium and Salmonella Mbandaka $(p<0.05)$. This difference correlated with the onset of lay. No Salmonella was detected in uninfected birds over the course of the experiment.

\section{Quantification of Salmonella in fecal samples using a serovar specific qPCR}

A quantitative PCR was developed to detect total Salmonella Typhimurium in single infection fecal samples and differentiate between Salmonella Typhimurium and Salmonella Mbandaka co-infection samples. A standard curve was generated by spiking uninfected, control feces spiked with known quantities of Salmonella Typhimurium. A cut-off $\mathrm{Ct}$ of 32 was used to exclude the detection of false positives and corresponded to $100 \mathrm{CFU}$ of 
Salmonella. For fecal samples spiked with both Salmonella Typhimurium and Salmonella Mbandaka, a cut-off $\mathrm{Ct}$ of 33 was used to exclude the detection of false positives. A Ct of 33 represented $1000 \mathrm{CFU}$ of Salmonella. Data are presented as mean $\log _{10} \mathrm{CFU} /$ gram feces \pm standard error of the mean.

The number of Salmonella detected by qPCR varied significantly in both treatment groups over the course of the experiment (Figure 2). The greatest amount of Salmonella detected in all groups was observed at week $1 \mathrm{pi}$ (Figure 2) with Salmonella Mbandaka in the co-infection group exhibiting the highest mean $\log _{10} \mathrm{CFU} /$ gram feces $(8.13 \pm 0.65)$. Interestingly, Salmonella Mbandaka had the highest mean $\log _{10}$ CFU/gram feces between weeks 1 through $14 \mathrm{pi}$, though this difference was not significant than Salmonella Typhimurium. After week 1, Salmonella detection was relatively stable and consistent and did not vary significantly. No significant correlation was observed between MPN counts and qPCR results.

\section{Fecal corticosterone metabolites in dried fecal extracts}

Measuring fecal corticosterone metabolites (FCM) is a non-invasive method enabling the measurement of one stress parameter [29, 30]. It has been previously shown that during point of lay, birds experience increased physiological stress and are thought to be immunocompromised [31]. Infection, however, has also been shown to affect plasma corticosterone levels [12]. Therefore, it was hypothesized that corticosterone should increase in all

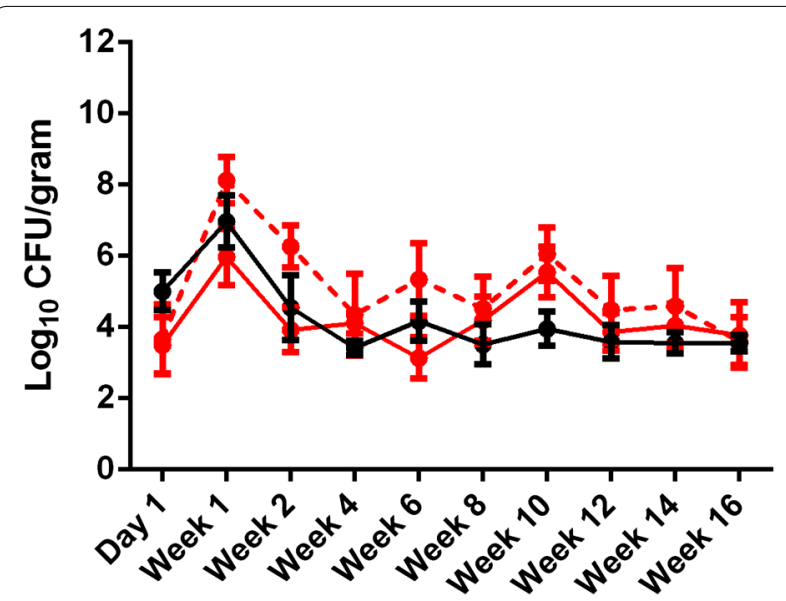

Figure 2 Quantification and differentiation of Salmonella by qPCR. Bacterial loads of fecal samples collected from birds infected with Salmonella Typhimurium only (black line) or co-infected with Salmonella Typhimurium (red line) and Salmonella Mbandaka (red hashed line) were quantified using a serovar specific QPCR. Salmonella Typhimurium was detected using a primers designed to the TSR3 gene while Salmonella Mbandaka was detected using the dhrfV gene. Data are presented as mean $\log _{10}$ CFU/gram feces \pm SEM. The amount of bacteria varied significantly over time $(p<0.01)$. chickens around the onset of lay, and infection may lead to further increase in level of corticosterone.

Fecal samples collected for enumeration of bacteria were also processed for FCM. A significant effect of time $(p<0.05)$ and treatment $(p<0.001)$ were observed between FCM concentrations (Figure 3). At day 1 pi, the FCM in birds infected with Salmonella Typhimurium $(674.2 \pm 109.3 \mathrm{pg} / \mathrm{mg})$ was significantly higher than the FCM observed for control birds $(238.0 \pm 12.62 \mathrm{pg} /$ $\mathrm{mg}$ ) or birds infected with a mixed inoculum of both Salmonella Typhimurium and Salmonella Mbandaka $(175.4 \pm 8.58 \mathrm{pg} / \mathrm{mg})(p<0.001)$.

At week $6 \mathrm{pi}$, the mean FCM $(625.2 \pm 113.2 \mathrm{pg} / \mathrm{mg})$ increased in birds co-infected with both Salmonella Typhimurium and Salmonella Mbandaka. At this time point, no significant difference between the two infection groups was detected. The mean FCM in control birds (268.7 $\pm 24.19 \mathrm{pg} / \mathrm{mg})$, however, was significantly less than both treatment groups $(p<0.01)$. At weeks $8,12,14$ and $16 \mathrm{pi}$, the mean FCM obtained for all groups varied but did not differ significantly (Figure 3 ).

No significant correlation was detected between the mean FCM concentration and MPN counts in singly or co-infected birds $\left(\mathrm{r}^{2}=-0.036, p=0.699\right)$. A significant but weak positive correlation $\left(\mathrm{r}^{2}=0.26, p=0.02\right)$ was observed between the mean log copy number/gram and FCM concentration in birds infected with Salmonella Typhimurium only.

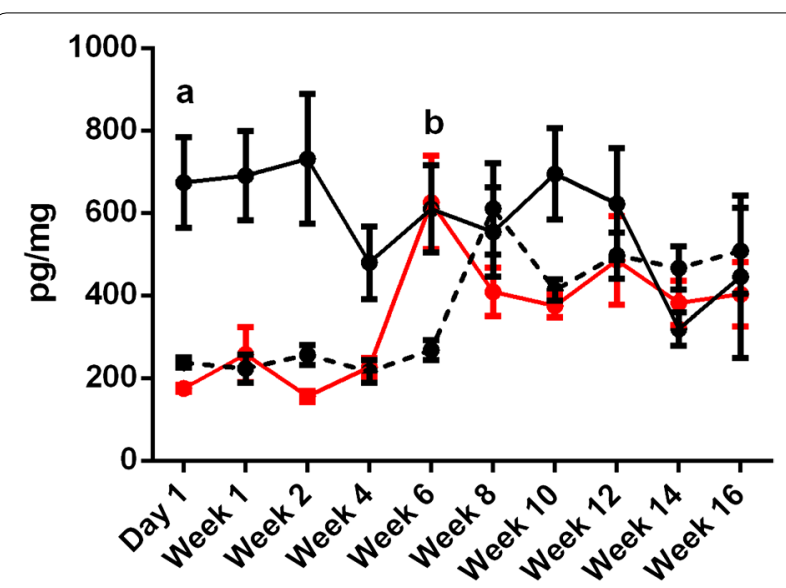

Figure 3 Measurement of fecal corticosterone metabolites (FCM). FCM concentrations were measured at day 1 and weeks $1,2,4,6,8,10,12,14$, and 16 pi. Data is presented as mean $\mathrm{pg} / \mathrm{mg}$ feces \pm SEM. A significant effect of time $(p<0.05)$ and treatment $(p<0.001)$ was detected for FCM concentrations. At day 1 pi, the mean FCM in birds infected with Salmonella Typhimurium (black line) was significantly higher than either control (black hashed line) or coinfected birds (red line) (a; $p<0.001)$. At week 6 pi, the mean FCM in co-infected birds increased. At this time point, both infection groups were exhibited significantly higher FCM concentrations than control birds $(b ; p<0.01)$. 
Detection of Salmonella from eggshell wash and internal contents

Eggs were collected at weeks $6,8,10,12$, and 14 pi and tested for the presence of Salmonella on the shell surface and within the internal contents. Salmonella was isolated throughout the experiment from the eggshell wash of experimentally infected hens. In birds infected with only Salmonella Typhimurium, the percentage of eggshell contamination ranged from 9.52 to $21.74 \%$. Birds infected with both Salmonella Typhimurium and Salmonella Mbandaka exhibited a similar level of eggshell contamination, 10.89-33.33\% (Table 1). By culture methods, the percentage of eggshell contamination was highest in both the groups at week 6 pi (onset of lay). No significant difference in eggshell contamination frequency was detected between Salmonella infection treatment groups. PCR results of egg shell samples indicated that the recovery rate of Salmonella Typhimurium (11.74\%) was higher than Salmonella Mbandaka (6.60\%) in co-infection group (Table 1).

No linear correlation was observed between the Salmonella MPN count in feces and eggshell contamination of infected birds $\left(\mathrm{r}^{2}=0.001, p=0.99\right)$. Salmonella was not detected in egg internal contents of either infection treatment group at any point during this experiment. Eggshells and internal contents from control hens were also negative for Salmonella.

Salmonella Typhimurium antibody titres in serum and egg yolk samples

The titres of Salmonella Typhimurium specific serum and yolk antibodies were measured over the course of the experiment (Figures $4 \mathrm{~A}$ and $\mathrm{B}$ ). The lowest mean antibody titre (antilog) in birds infected with only Salmonella Typhimurium was observed at week 1 pi (1286 \pm 168.1$)$ and peaked at week 6 pi (2678 \pm 179.5$)$. After week 6 pi, antibody titres remained constant during the remainder of the experiment. A similar pattern was observed for Salmonella Typhimurium antibodies measured from the co-infection group. In birds infected with both Salmonella Typhimurium and Salmonella Mbandaka, the mean titre was lowest at week 1 pi $(997.7 \pm 170.5)$ and highest at week 6 pi (1949 \pm 239.1$)$. Mean antibody titres of birds infected with Salmonella Typhimurium only were significantly higher than those obtained for the co-infection group at weeks $6,8,10,12$, and $14 \mathrm{pi}(p<0.01)$. Control birds were negative for Salmonella Typhimurium antibodies over the course of the experiment.

Eggs collected from both infection groups tested positive for Salmonella yolk antibodies (Figure 4B). A significant effect of treatment was detected between the experimental groups $(p \leq 0.01)$.

\section{Persistence of Salmonella in internal organs}

At 30 weeks of age (week 16 pi), the experiment was terminated and birds were euthanized. Spleen, liver, bone marrow and caecal tonsils from all hens were collected and processed for Salmonella to characterise the persistence of the bacteria in these organs. All samples collected from control hens were negative for Salmonella. Bacteria were detected in all tissues except for the bone marrow samples. The total number of positive samples was greatest in the spleen, followed by the liver and caecal tonsils (Figure 5). The mean splenic bacterial load observed in birds infected with only Salmonella Typhimurium (757.4 $\pm 301.1 \mathrm{CFU} / \mathrm{g}$ tissue) was significantly greater than the mean titre observed for birds inoculated with both Salmonella Typhimurium and Salmonella Mbandaka (236.0 $\pm 54.51 \mathrm{CFU} / \mathrm{g}$ tissue) $(p<0.01)$.

Birds infected with both Salmonella Typhimurium and Salmonella Mbandaka exhibited the highest number of individuals positive for Salmonella in the liver

Table 1 Percentage of isolation of Salmonella and Salmonella Typhimurium by culture and PCR method respectively from eggshell samples of orally infected birds at different weeks of pi

\begin{tabular}{|c|c|c|c|c|c|}
\hline \multirow[t]{2}{*}{ Weeks pi } & \multicolumn{2}{|c|}{ Salmonella Typhimurium only group } & \multicolumn{3}{|l|}{ Co-infection group } \\
\hline & $\begin{array}{l}\text { Salmonella detection } \\
\text { by culture method }\end{array}$ & $\begin{array}{l}\text { Salmonella Typhimu- } \\
\text { rium detection by PCR }\end{array}$ & $\begin{array}{l}\text { Salmonella detection } \\
\text { by culture method }\end{array}$ & $\begin{array}{l}\text { Salmonella Typhimu- } \\
\text { rium detection by PCR }\end{array}$ & $\begin{array}{l}\text { Salmonella Mbandaka } \\
\text { detection by PCR }\end{array}$ \\
\hline Week 6 & $21.74^{c}(5 / 23)^{a}$ & $17.39(4 / 23)$ & $33.33(8 / 24)$ & $8.33(2 / 24)^{b}$ & $8.33(2 / 24)^{b}$ \\
\hline Week 8 & $9.52(8 / 84)$ & $8.33(7 / 84)$ & $10.89(11 / 101)$ & $8.91(9 / 101)$ & $0.00(0 / 101)$ \\
\hline Week 10 & $15.85(13 / 82)$ & $15.85(13 / 82)$ & $15.22(14 / 92)$ & $10.87(10 / 92)$ & $5.43(5 / 92)$ \\
\hline Week 12 & $13.48(12 / 89)$ & $12.36(11 / 89)$ & $22.92(22 / 96)$ & $14.58(14 / 96)$ & $13.54(13 / 96)$ \\
\hline Week 14 & $11.49(10 / 87)$ & $10.34(9 / 87)$ & $21.88(21 / 96)$ & $13.54(13 / 96)$ & $7.29(7 / 96)$ \\
\hline Total & $13.15(48 / 365)$ & $12.05(44 / 365)$ & $13.20(54 / 409)$ & $11.74(48 / 409)$ & $6.60(27 / 409)$ \\
\hline
\end{tabular}

\footnotetext{
a Number of positive eggs/total number of eggs tested.

b Results confirmed by PCR.

c Values in \%.
} 

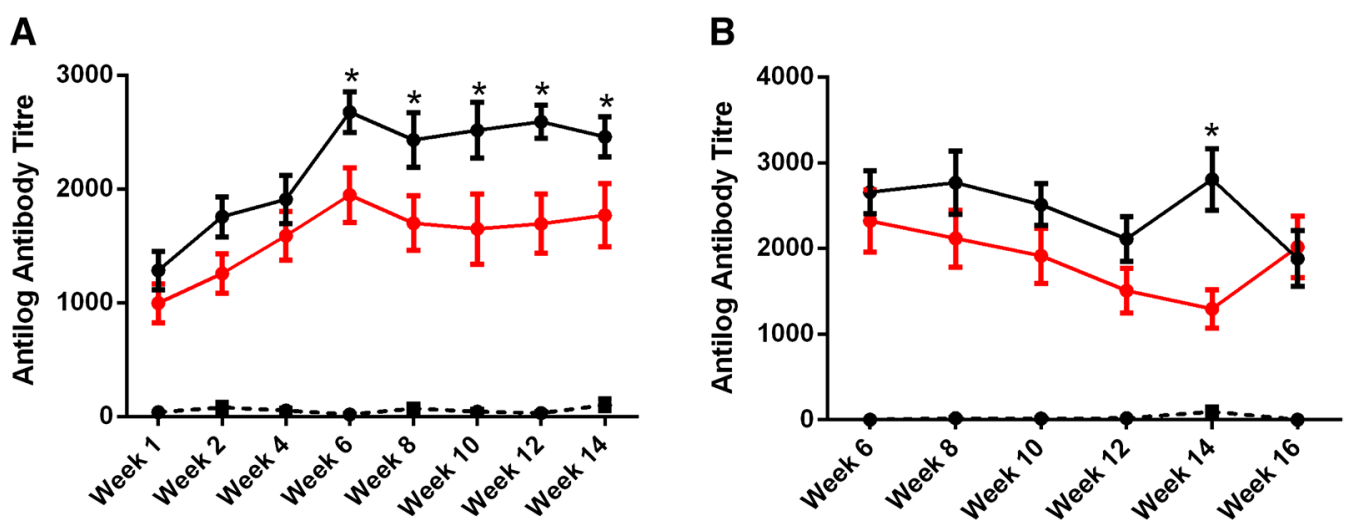

Figure 4 Quantification of Salmonella Typhimurium specific antibodies in serum and yolk. Salmonella Typhimurium specific antibody titers (antilog antibody titres \pm SEM) were characterised over the course of the experiment in both serum $(\mathbf{A})$ and yolk (B) in control birds (black hashed line) as well as hens infected with only Salmonella Typhimurium (black line) or a combination of both Salmonella Typhimurium and Salmonella Mbandaka (red line). In the serum, the amount of Salmonella Typhimurium antibody was significantly higher in singly infected birds compare with co-infected birds from week 6 pi till the end of the experiment $\left({ }^{*} p<0.01\right)$. Mean antibody titres detected in yolk samples collected from Salmonella Typhimurium infected birds were only significantly different from co-infected birds at week 14 pi $\left({ }^{*} p<0.01\right)$.

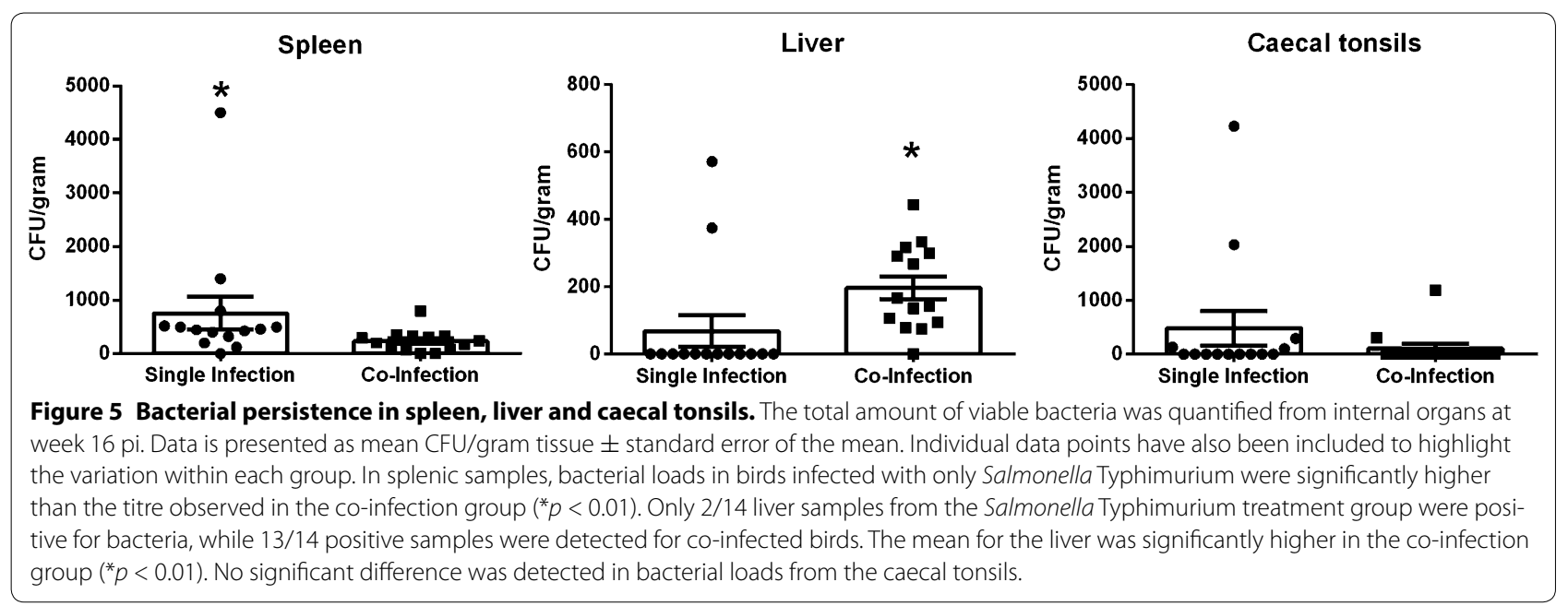

(92.9\%) (Figure 5). The mean bacterial titre for the coinfection group was $197.1 \pm 34.17 \mathrm{CFU} / \mathrm{g}$ tissue and was significantly higher than the mean titre observed for birds infected with Salmonella Typhimurium, $68.46 \pm 46.97 \mathrm{CFU} / \mathrm{g}$ tissue (Mann-Whitney, $p<0.01$ ). The lowest overall level of Salmonella colonisation was observed in the caecal tonsils with $35.7 \%$ positive individuals in the single infection group and $14.3 \%$ positive birds in the multi-serovar group (Figure 5). Bacterial titres in caecal tonsils collected from birds infected with only Salmonella Typhimurium ranged from 0 to $4.2 \times 10^{3} \mathrm{CFU} / \mathrm{g}$ tissue with a mean titre of $485.7 \pm 321.9 \mathrm{CFU} / \mathrm{g}$ tissue. Birds infected with both Salmonella Typhimurium and Salmonella Mbandaka ranged from 0 to $1.2 \times 10^{3} \mathrm{CFU} / \mathrm{g}$ tissue with a mean of $107.1 \pm 85.79 \mathrm{CFU} / \mathrm{g}$ tissue. No significant difference was detected between the two infection treatment groups.

The culture positive internal organs were further tested by PCR to differentiate Salmonella Typhimurium and Salmonella Mbandaka in the co-infection group (Table 2). In splenic samples, 1 of 14 was positive for Salmonella Typhimurium while 4/14 samples were positive for Salmonella Mbandaka. Three of 14 liver samples from the co-infection group were positive for Salmonella Mbandaka, however, no Salmonella Typhimurium was detected. In the caecal tonsils, $1 / 14$ samples were positive for Salmonella Typhimurium and 1/14 tested positive for Salmonella Mbandaka. 


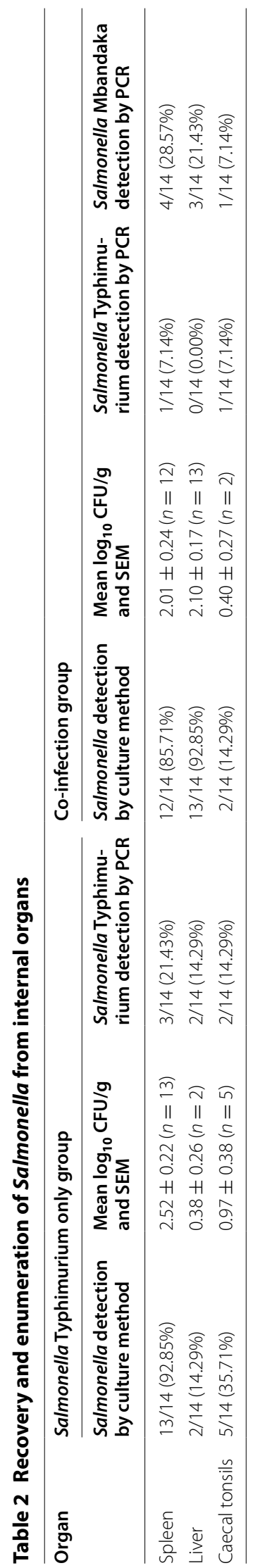




\section{Discussion}

This study indicated that layers infected with Salmonella Typhimurium DT9 became persistently infected causing intermittent bacterial shedding in faeces. At week 6 pi, the MPN count in Salmonella Typhimurium infected group was significantly higher than multi-serovar infection group. Week 6 corresponded to the onset of lay in experimental birds and it was postulated that this increase could be related to physiological stress induced by onset of lay [16].

FCM levels in the Salmonella Typhimurium group were higher than either the control or multi-serovar treatment groups from day 1 until week 6 pi. Bacterial lipopolysaccharide (LPS) can induce inflammation within a host and has been associated with increased serum and corticosterone [12]. The LPS of Salmonella enterica is variable, serovar dependent and contributes to different degrees of virulence. This may account for lower mean FCM concentrations in the multi-serovar group. Increase in FCM in all treatment groups between 6-8 weeks could be attributed to the onset of lay, and infection may lead to further increase in level of FCM. However, it important to note that no positive correlation was observed between bacterial shedding and FCM levels in this study.

During this study, higher rates of eggshell contamination at the onset of lay could be attributed to increased Salmonella shedding in feces at that point $[5,16]$. There was no linear correlation between Salmonella shedding in feces and egg shell contamination of infected birds and this is in agreement with earlier reports [32]. Of note, in this study Salmonella was not detected from egg internal contents.

The Salmonella Typhimurium IgG antibody titres increased after week 1 pi and peaked at week 6 pi. Birds were seropositive till the end of the trial at week 14 pi but the immune response did not result in complete clearance of Salmonella spp. It is also important to note that the antibody response contributes to the clearance of extracellular bacteria, intracellular bacteria can persist in the host thus cell mediated immune response is essential for clearance of Salmonella Typhimurium (reviewed in [33]). Overall decreased IgG antibody response in multiserovar infection group could perhaps be due to the competitive and immunoprotective mechanism between both Salmonella strains. However, the absence of an infection treatment with only Salmonella Mbandaka in this study limits this conclusion.

In the multi-serovar group, a low MPN was obtained at week 6 yet qPCR results revealed similar loads of both Salmonella Typhimurium and Salmonella Mbandaka. The discrepancy may be due to the detection of both live and dead bacteria using PCR method. However it is unclear why that has happened specifically at week 6 pi.
Laying hens mounted immune response to invasive strain of Salmonella Typhimurium DT9 without inducing clinical signs. Variation in qPCR and MPN results could be attributed to the sensitivity of the tests used. Several factors such as heterogeneous distribution of the pathogen in sample, number of stressed cells, sample matrix, enrichment time and enrichment media can influence the accuracy of quantitation [34].

In Salmonella Typhimurium infected group there were increased levels of FCM concentrations, antibody titres and bacterial shedding (as detected by MPN method) at week $6 \mathrm{pi}$ (onset of lay) which supports the theory that the presence of stress hormones can stimulate Salmonella growth and enhance bacterial colonisation in the intestine [35]. However present data suggests that this theory may not apply when host is infected with multiple Salmonella serovars. Concentration of corticosterone levels in sera can increase or decrease the antibody response [36]. In this study the high corticosterone levels did not suppress the humoral immune response against Salmonella Typhimurium.

Stress can stimulate the recrudescence of bacteria from internal organs resulting in high bacterial load in feces [37]. Our findings indicated that Salmonella Typhimurium persisted in internal organs despite high levels of circulating specific IgG antibody. Previous studies reported Salmonella Typhimurium clearance from liver and other internal organs due to Th-1 dominated responses and high levels of interferon- $\gamma$ expression at around 14-28 days pi [38]. Some studies reported low frequency of Salmonella Enteritidis in liver and other internal organs for up to 22 weeks pi [39]. It has also been suggested that age at exposure did not affect recovery of Salmonella Typhimurium from liver [40]. Our observations could not be compared with previous reports because such studies were performed using broilers for short period of time. It could be hypothesised that persistence of Salmonella Typhimurium in internal organs including liver could be due to the timing of challenge (prior to lay in this case). Sexual maturity can induce immunosuppression by altering cellular and humoral immune response [33]. This could ultimately cause bacteria to avoid clearance and dominate host leading to a recrudescence of infection. However further studies are required to confirm this hypothesis. It is interesting to note that in mixed infection group, only Salmonella Mbandaka was detected from liver.

Previous literature stated that intestinal persistence of Salmonella Typhimurium in chickens was longer when birds were challenged at day old compared to day 7 and that older birds are considerably more resistant to salmonellae than are young chicks $[41,42]$. Our study indicated that infection of adult birds (14 weeks old in this study) 
can also result in continued harbouring of the Salmonella Typhimurium and intermittent faecal shedding. This shedding can be associated with the stress event such as onset of lay. However interplay between stress, immune response and Salmonella Typhimurium shedding in single or mixed infection group at the onset of lay is more complex to understand.

To conclude, Salmonella Typhimurium DT9 persistently infected hens causing intermittent bacterial shedding in faeces. At the onset of lay shedding of Salmonella Typhimurium was affected in mixed infection group. Increased immune response did not result in clearance of Salmonella spp (except for Salmonella Typhimurium at week 6 pi). There was no correlation between FCM and Salmonella shedding. This long term Salmonella Typhimurium infection model provided useful insights on the continued persistence and or recrudescence of Salmonella Typhimurium, although further investigation is necessary to understand the immunobiology of long term and systemic Salmonella Typhimurium infection.

\section{Competing interests}

The authors declare that they have no competing interests.

\section{Authors' contributions}

$P S, V P, A M$ and $K C$ designed the experiments and were involved with preparation of this manuscript. PS, VP, AM and KC conducted the layer hen trial. PS performed all sampling and microbiological processing. TM performed corticosterone extractions from fecal samples. PS and TM conducted FCM experiments. TM and KC performed qPCR. All authors read and approved the final manuscript.

\section{Acknowledgements}

We thank Associate Professor Milton McAllister, Dr. Vaibhav Gole and Dr. Rebecca Forder for their assistance with this study.

\section{Funding}

This research work was supported by Australian Egg Corporation Limited (AECL) Australia. Mr. Pardeep Sharma is a recipient of an International Postgraduate Research Scholarship from the University of Adelaide Australia.

Received: 11 November 2016 Accepted: 6 January 2017

Published online: 06 February 2017

\section{References}

1. Moffatt CR, Musto J, Pingault N, Miller M, Stafford R, Gregory J, Polkinghorne BG, Kirk MD (2016) Salmonella Typhimurium and outbreaks of egg-associated disease in Australia, 2001 to 2011. Foodborne Pathog Dis 13:379-385

2. Threlfall E, Wain J, Peters T, Lane C, De Pinna E, Little C, Wales A, Davies R (2014) Egg-borne infections of humans with salmonella: not only an $S$. Enteritidis problem. World Poultr Sci J 70:15-26

3. Chousalkar KK, Sexton M, McWhorter A, Hewson K, Martin G, Shadbolt C, Goldsmith P (2015) Salmonella Typhimurium in the Australian egg industry: multidisciplinary approach to addressing the public health challenge and future directions. Crit Rev Food Sci Nutr. doi:10.1080/10408398.2015. 1113928

4. Williams A, Davies A, Wilson J, Marsh P, Leach S, Humphrey T (1998) Contamination of the contents of intact eggs by Salmonella Typhimurium DT104. Vet Rec 143:562-563
5. Okamura M, Sonobe M, Obara S, Kubo T, Nagai T, Noguchi M, Takehara K, Nakamura M (2010) Potential egg contamination by Salmonella enterica serovar Typhimurium definitive type 104 following experimental infection of pullets at the onset of lay. Poult Sci 89:1629-1634

6. Okamura M, Miyamoto T, Kamijima Y, Tani H, Sasai K, Baba E (2001) Differences in abilities to colonize reproductive organs and to contaminate eggs in intravaginally inoculated hens and in vitro adherences to vaginal explants between Salmonella Enteritidis and other Salmonella serovars. Avian Dis 45:962-971

7. Leach SA, Williams A, Davies AC, Wilson J, Marsh PD, Humphrey TJ (1999) Aerosol route enhances the contamination of intact eggs and muscle of experimentally infected laying hens by Salmonella Typhimurium DT104. FEMS Microbiol Lett 171:203-207

8. Borsoi A, Quinteiro-Filho WM, Calefi AS, Piantino Ferreira AJ, AstolfiFerreira CS, Florio JC, Palermo-Neto J (2015) Effects of cold stress and Salmonella Heidelberg infection on bacterial load and immunity of chickens. Avian Pathol 44:490-497

9. Quinteiro-Filho WM, Rodrigues M, Ribeiro A, Ferraz-de-Paula V, Pinheiro M, Sa L, Ferreira A, Palermo-Neto J (2012) Acute heat stress impairs performance parameters and induces mild intestinal enteritis in broiler chickens: role of acute hypothalamic-pituitary-adrenal axis activation. J Anim Sci 90:1986-1994

10. Holt PS (1993) Effect of induced molting on the susceptibility of White Leghorn hens to a Salmonella Enteritidis infection. Avian Dis 37:412-417

11. Nakamura M, Nagamine N, Takahashi T, Suzuki S, Kijima M, Tamura Y, Sato S (1994) Horizontal transmission of Salmonella Enteritidis and effect of stress on shedding in laying hens. Avian Dis 38:282-288

12. Shini S, Kaiser P, Shini A, Bryden WL (2008) Biological response of chickens (Gallus gallus domesticus) induced by corticosterone and a bacterial endotoxin. Comp Biochem Physiol B: Biochem Mol Biol 149:324-333

13. Mashaly M, Hendricks G, Kalama M, Gehad A, Abbas A, Patterson P (2004) Effect of heat stress on production parameters and immune responses of commercial laying hens. Poult Sci 83:889-894

14. Quinteiro-Filho W, Gomes A, Pinheiro M, Ribeiro A, Ferraz-de-Paula V, Astolfi-Ferreira C, Ferreira A, Palermo-Neto J (2012) Heat stress impairs performance and induces intestinal inflammation in broiler chickens infected with Salmonella Enteritidis. Avian Pathol 41:421-427

15. Gole VC, Torok V, Sexton M, Caraguel CG, Chousalkar KK (2014) Association between indoor environmental contamination by Salmonella enterica and contamination of eggs on layer farms. J Clin Microbiol 52:3250-3258

16. Gole VC, Caraguel CG, Sexton M, Fowler C, Chousalkar KK (2014) Shedding of Salmonella in single age caged commercial layer flock at an early stage of lay. Int J Food Microbiol 189:61-66

17. Pulido-Landínez M, Sanchez-Ingunza R, Guard J, Nascimento V (2013) Assignment of serotype to Salmonella enterica isolates obtained from poultry and their environment in Southern Brazil. Lett Appl Microbiol 57:288-294

18. Chousalkar K, Gole V, Caraguel C, Rault JL (2016) Chasing Salmonella Typhimurium in free range egg production system. Vet Microbiol 192:67-72

19. Glass K, Fearnley E, Hocking H, Raupach J, Veitch M, Ford L, Kirk MD (2016) Bayesian source attribution of salmonellosis in South Australia. Risk Anal 36:561-570

20. Doyle EM (2013) White paper on human illness caused by Salmonella from all food and non-food vectors. Update 2013 in: FRI Food Safety Reviews (Ed), Madison, pp 1-45

21. Guard J, Sanchez-Ingunza R, Shah DH, Rothrock MJ, Gast RK, Jones DR (2015) Recovery of Salmonella enterica serovar Enteritidis from hens initially infected with serovar Kentucky. Food Chem 189:86-92

22. Pulido-Landínez M, Sanchez-Ingunza R, Guard J, do Nascimento VP (2013) Presence of Salmonella Enteritidis and Salmonella Gallinarum in commercial laying hens diagnosed with fowl typhoid disease in Colombia. Avian Dis 58:165-170

23. Pande W, Devon RL, Sharma P, McWhorter AR, Chousalkar KK (2016) Study of Salmonella Typhimurium infection in laying hens. Front Microbiol 7:203

24. USDA, U.S. Department of agriculture, food safety and inspection services (2008) most probable number procedure and tables. In: Microbiology Laboratory Guidebook. https://www.fsis.usda.gov/wps/wcm/ 
connect/8872ec11-d6a3-4fcf-86df-4d87e57780f5/MLG-Appendix-2. pdf?MOD=AJPERES. Accessed 14 Jan 2015

25. Pande V, Gole VC, McWhorter AR, Abraham S, Chousalkar KK (2015) Antimicrobial resistance of non-typhoidal Salmonella isolates from egg layer flocks and egg shells. Int J Food Microbiol 203:23-26

26. Akiba M, Kusumoto M, Iwata T (2011) Rapid identification of Salmonella enterica serovars, Typhimurium, Choleraesuis, Infantis, Hadar, Enteritidis, Dublin and Gallinarum, by multiplex PCR. J Microbiol Methods 85:9-15

27. Gole V, Chousalkar K, Roberts J (2012) Prevalence of antibodies to Mycoplasma synoviae in laying hens and possible effects on egg shell quality. Prev Vet Med 106:75-78

28. Greiner M, Sohr D, Gobel P (1995) A modified ROC analysis for the selection of cut-off values and the definition of intermediate results of serodiagnostic tests. J Immunol Methods 185:123-132

29. Dehnhard M, Schreer A, Krone O, Jewgenow K, Krause M, Grossmann R (2003) Measurement of plasma corticosterone and fecal glucocorticoid metabolites in the chicken (Gallus domesticus), the great cormorant (Phalacrocorax carbo), and the goshawk (Accipiter gentilis). Gen Comp Endocrinol 131:345-352

30. Rettenbacher S, Mostl E, Hackl R, Ghareeb K, Palme R (2004) Measurement of corticosterone metabolites in chicken droppings. Br Poult Sci 45:704-711

31. Shini S, Huff G, Shini A, Kaiser P (2010) Understanding stress-induced immunosuppression: exploration of cytokine and chemokine gene profiles in chicken peripheral leukocytes. Poult Sci 89:841-851

32. Gast RK, Guard-Bouldin J, Holt PS (2005) The relationship between the duration of fecal shedding and the production of contaminated eggs by laying hens infected with strains of Salmonella Enteritidis and Salmonella Heidelberg. Avian Dis 49:382-386

33. Chappell L, Kaiser P, Barrow P, Jones MA, Johnston C, Wigley P (2009) The immunobiology of avian systemic salmonellosis. Vet Immunol Immunopathol 128:53-59
34. Malorny B, Lofstrom C, Wagner M, Kramer N, Hoorfar J (2008) Enumeration of Salmonella bacteria in food and feed samples by real-time PCR for quantitative microbial risk assessment. Appl Environ Microbiol 74:1299-1304

35. Verbrugghe E, Dhaenens M, Leyman B, Boyen F, Shearer N, Van Parys A, Haesendonck R, Bert W, Favoreel H, Deforce D, Thompson A, Haesebrouck F, Pasmans F (2016) Host stress drives Salmonella recrudescence. Sci Rep 6:20849

36. Campos-Rodriguez R, Kormanovski A, Stephano AQ, Abarca-Rojano E, Berczi I, Ventura-Juarez J, Drago-Serrano ME (2012) The central nervous system modulates the immune response to Salmonella. In: Kumar Yashwant (ed) Salmonella — a diversified superbug. Intech Open Access Publisher, Croatia, pp 375-398

37. Rostagno M, Wesley I, Trampel D, Hurd H (2006) Salmonella prevalence in market-age turkeys on-farm and at slaughter. Poult Sci 85:1838-1842

38. Beal RK, Powers C, Wigley P, Barrow PA, Smith AL (2004) Temporal dynamics of the cellular, humoral and cytokine responses in chickens during primary and secondary infection with Salmonella enterica serovar Typhimurium. Avian Pathol 33:25-33

39. Gast RK, Beard CW (1990) Isolation of Salmonella enteritidis from internal organs of experimentally infected hens. Avian Dis 34:991-993

40. Gast RK, Beard CW (1989) Age-related changes in the persistence and pathogenicity of Salmonella Typhimurium in chicks. Poult Sci 68:1454-1460

41. Groves PJ, Sharpe SM, Muir WI, Pavic A, Cox JM (2016) Live and inactivated vaccine regimens against caecal Salmonella Typhimurium colonisation in laying hens. Aust Vet J 94:387-393

42. Gast RK (2008) Paratyphoid infections. In: Saif Y (Ed) Diseases of Poultry, 12th edn. Blackwell, Ames, pp 636-655

\section{Submit your next manuscript to BioMed Central and we will help you at every step:}

- We accept pre-submission inquiries

- Our selector tool helps you to find the most relevant journal

- We provide round the clock customer support

- Convenient online submission

- Thorough peer review

- Inclusion in PubMed and all major indexing services

- Maximum visibility for your research

Submit your manuscript at www.biomedcentral.com/submit
() Biomed Central 